\title{
A nicotina atua como fator deletério na reparação da parede abdominal ${ }^{1}$
}

\author{
Aldo da Cunha Medeiros ${ }^{2}$ \\ Francisco Pignataro Lima ${ }^{3}$ \\ Antônio Medeiros Dantas Filho ${ }^{4}$ \\ Nara Medeiros Cunha de Melo ${ }^{5}$ \\ Ítalo Medeiros de Azevêdo ${ }^{6}$
}

\begin{abstract}
Medeiros AC, Lima FP, Dantas Filho AM, Melo NMC, Azevêdo IM. A nicotina atua como fator deletério na reparação da parede abdominal. Acta Cir Bras [online] 2003 vol 18 suppl 1. Disponível em www.scielo.br/acb.
\end{abstract}

RESUMO: Objetivo: Avaliar o efeito da nicotina na cicatrização da camada musculoaponeurótica da parede abdominal. Métodos: Estudo experimental em que foram usados 16 ratos da raça Wistar pesando em média $210 \pm 8 \mathrm{~g}$, separados aleatoriamente em 2 grupos de 8 . Nos animais do grupo A foi implantado disco de nicotina (Nicotinel Ò) na dose de $5 \mathrm{mg} / \mathrm{Kg}$ de peso/dia no subcutâneo da região dorsal, trocado a cada dois dias, a partir do $5^{\circ}$ dia antes da operação em que foi feita laparotomia mediana de $5 \mathrm{~cm}$, até o $10^{\circ}$ dia de observação. No grupo B (controle) foram usados discos de celulose com o mesmo diâmetro. Tubo de silicone multiperfurado foi implantado no subcutâneo a $1 \mathrm{~cm}$ da lesão da parede abdominal. A camada musculoaponeurótica e a pele foram suturadas com fio de nylon $5-0$. No $10^{\circ}$ dia pós-operatório foi colhido $1 \mathrm{ml}$ de líquido seroso do tubo de silicone por punção percutânea para dosagem de $\mathrm{pO}_{2}$ e os animais receberam dose letal de anestésico. Foi ressecado um segmento da camada musculoaponeurótica com $2 \mathrm{~cm}$ de largura para tensiometria, em seguida processado e corado em HE e tricrômico de Masson para análise quantitativa dos dados histopatológicos em sistema digitalizado. A análise estatística foi feita pelo ANOVA e teste Newman-Keuls, com significância 0,05 . Resultados: No grupo $\mathrm{A}$ a $\mathrm{pO}_{2}$ do líquido tecidual atingiu o valor $17,75 \pm 3,4 \mathrm{mmHg}$ e no grupo $\mathrm{B}$ (controle) a $\mathrm{pO}_{2}=40,75 \pm 6,4 \mathrm{mmHg}(\mathrm{p}<0,01)$. A

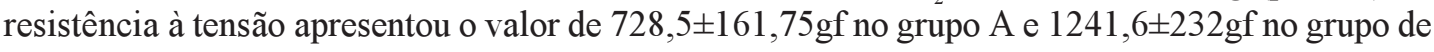
controle $(\mathrm{p}<0,01)$, coincidindo com os achados da $\mathrm{pO}_{2}$ A densidade média dos elementos histopatológicos estudados foi de $105 \pm 17,1$ nos animais do grupo A e $146,2 \pm 8,8$ no grupo $B$ ( $<<0,01$ ). Conclusão: após avaliação da $\mathrm{pO}_{2}$ tecidual, tensiometria e histopatologia, concluiu-se que a nicotina por via subcutânea exerce efeito deletério sobre a cicatrização de lesões da parede abdominal de ratos.

DESCRITORES: Nicotina. Cicatrização. Parede abdominal. Tensiometria. Histopatologia. Oxigênio.

1. Trabalho realizado no Núcleo de Cirurgia Experimental Prof. Travassos Sarinho -UFRN. Apoiado pelo CNPq.

2. Doutor em Cirurgia, Chefe do Núcleo de Cirurgia Experimental, Prof. Adjunto e Coordenador da Disciplina de Técnica Operatória -UFRN, Pesquisador nível I do CNPq.

3. Mestre e Prof. do Departamento de Patologia -UFRN.

4. Prof. do Departamento de Cirurgia e aluno do Programa de Pós-graduação-Doutorado.

5. Aluna bolsista de Iniciação Científica.

6. Aluno do Departamento de Estatística-UFRN. 


\section{INTRODUÇÃO}

O tabaco é considerado como droga pelas normas que regem o "U.S Food and Drug Administration", que o inclui no mesmo grupo de substâncias como morfina, nicotina, ópio, entre muitas outras que causam dependência.

São bem conhecidos os efeitos negativos do cigarro no aparelho respiratório, provocando doenças como enfisema e a bronquite, bem como o elevado índice de câncer do pulmão e de outros órgãos relacionados ao consumo de cigarro ${ }^{1,2}$. No que diz respeito à cirurgia, não é novo o conceito de que o ato de fumar prejudica a cicatrização das feridas. Os fumantes apresentam um maior risco de deiscência das feridas abdominais $^{3}$, necrose de enxertos de pele ${ }^{4,5}$ e cicatrização precária de úlceras de perna ${ }^{6}$. Em um estudo de cicatrização de feridas em diabéticos fumantes, Goodson et $\mathrm{al}^{7}$ relataram uma deposição deficiente de colágeno.

$\mathrm{O}$ estudo aqui descrito procurou investigar experimentalmente a ação da nicotina aplicada por via subcutânea, na resistência dos tecidos suturados, na $\mathrm{pO}_{2}$ dos tecidos em cicatrização e na evolução histopatológica desses tecidos da parede abdominal, após laparotomia mediana em ratos.

\section{MÉTODOS}

Foram utilizados 16 ratos adultos jovens da raça Wistar, com peso médio $210 \pm 8 \mathrm{~g}$, mantidos em gaiolas individuais com água e comida ad libitum.

No modelo animal utilizado foi feita a aplicação de nicotina, introduzindo-se o Nicotinell TTS ${ }^{\circledR}$ na dose de $5 \mathrm{mg} / \mathrm{Kg} /$ dia em dias alternados, em espaço subcutâneo cirurgicamente confeccionado no dorso dos animais. Foram operados em condições de assepsia e divididos aleatoriamente em dois grupos de igual número de animais, sendo os grupos $A(n=8)$ e $B(n=8)$, anestesiados com éter sulfúrico por inalação. Os animais do grupo A receberam a nicotina durante 6 dias antes da operação a que foram submetidos, para assegurar nível sérico da droga no momento da operação e por mais 10 dias no pós-operatório. No grupo B (controle) foram utilizados discos esterilizados de celulose sem nicotina, ao nível do dorso, na mesma localização e prazo de utilização do grupo experimental.

Em decúbito dorsal e devidamente anestesiados, todos os animais foram submetidos a depilação, antisepsia da região abdominal, laparotomia mediana de 5 $\mathrm{cm}$. A parede abdominal foi suturada em dois planos em pontos simples separados com fio de nylon monofilamentar 5-0. Um tubo de silicone multiperfurado, com $3 \mathrm{~mm}$ de diâmetro e $2 \mathrm{~cm}$ de comprimento foi implantado no subcutâneo próximo à zona de sutura. Completado o período de uso da droga antes descrito, procedeu-se a coleta de $1 \mathrm{ml}$ de líquido seroso da luz do tubo de silicone por punção percutânea, para determinação da pressão parcial do oxigênio $\left(\mathrm{pO}_{2}\right)$ e em seguida a morte dos animais com superdose de anestésico. Foi retirado segmento da parede abdominal incluindo a ferida da camada musculo-aponeurótica no sentido transversal, tendo a ferida no seu centro, com $2 \mathrm{~cm}$ de largura. A zona de sutura foi submetida a teste de resistência à tensão (em gf) com o auxílio da Máquina de Ensaios EMIC DL500MF interligada a programa específico versão 3.0 para Windows. Concluído o teste de resistência, foi feita biópsia da zona de sutura, sendo o material fixado em formol $10 \%$ e processado através de técnica de rotina usando-se as colorações hematoxilina-eosina e tricrômico de Masson, seguindo a técnica utilizada por outros autores ${ }^{8}$. A avaliação histológica das lâminas foi efetuada em microscópio óptico (Olimpus) equipado com sistema digital de capturada de imagens através de câmera e Software Image Pro-plus, versão 3.0 (Media Cybernetics - LP, USA). Cada campo digitalizado foi dividido em unidades de imagem denominadas picture elements ou pixels. Foram avaliados dez campos aleatórios de cada lâmina para quantificação dos elementos da reação inflamatória fibroblastos, neutrófilos, neovasos, tecido de granulação e colágeno, sob a forma de densidade mínima, máxima e média.

Os dados foram avaliados pela análise de variância ANOVA, complementada pelo exame das diferenças entre as médias através do teste Newman-Keuls, considerando-se as diferenças significantes quando $\mathrm{p}<0,05$.

\section{RESULTADOS}

Entre os animais do grupo A, dois deles (números 3 e 5) apresentaram pequeno grau de infecção da ferida operatória. $\mathrm{A} \mathrm{pO}_{2}$ do líquido tecidual próximo à lesão da parede abdominal atingiu a média de 17,75 $\pm 3,4$ $\mathrm{mmHg}$ no grupo submetido a tratamento com nicotina, no qual se observou que os animais de números 3 e 5 , acometidos de infecção da ferida operatória, apresentaram os menores valores de $\mathrm{pO}_{2}$. No grupo $\mathrm{B}$ (de controle) a $\mathrm{pO}_{2}$ correspondeu ao valor $40,75 \pm 6,4$ $\mathrm{mmHg}$. Na tabela 1 observa-se que quando foram comparadas as duas médias, a diferença mostrou-se significante $(p<0,01)$. 
Tabela 1 - Valores em mmHg da dosagem da $\mathrm{PO}_{2}$ do líquido seroso colhido da vizinhança do tecido músculoaponeurótico em cicatrização.

\begin{tabular}{c|c|c}
\hline${\text { Rato } \mathbf{~}^{\mathbf{0}}}$ & Grupo A (nicotina) & Grupo B (controle) \\
\hline 01 & 20 & 38 \\
\hline 02 & 17 & 44 \\
\hline 03 & 14 & 35 \\
\hline 04 & 18 & 51 \\
\hline 05 & 12 & 42 \\
\hline 06 & 18 & 37 \\
\hline 07 & 22 & 32 \\
\hline 08 & 21 & 47 \\
\hline Média $\pm \mathrm{dp}$ & $17,75 \pm 3,4$ & $40,75 \pm 6,4$ \\
\hline
\end{tabular}

A diferença entre as médias é significante $(\mathrm{P}<0,01)$

A resistência da camada musculo-aponeurótica à tensão apresentou o valor de $728,5 \pm 161,75 \mathrm{gf}$ nos animais do grupo experimental e $1241,6 \pm 232 \mathrm{gf}$ no grupo de controle $(\mathrm{p}<0,01)$, coincidindo com os achados da $\mathrm{pO}_{2}$ (tabela 2).

Tabela 2 - Resistência dos tecidos suturados à tensão, determinados em grama-força (gf).

\begin{tabular}{c|c|c}
\hline${\text { Rato } \mathbf{~}^{\mathbf{0}}}$ & Grupo A (nicotina) & Grupo B (controle) \\
\hline 01 & 745 & 1183 \\
\hline 02 & 598 & 978 \\
\hline 03 & 870 & 1493 \\
\hline 04 & 459 & 1620 \\
\hline 05 & 952 & 980 \\
\hline 06 & 627 & 1314 \\
\hline 07 & 842 & 1275 \\
\hline 08 & 737 & 1090 \\
\hline Média $\pm \mathrm{dp}$ & $728,5 \pm 161,75^{*}$ & $1241,6 \pm 232$ \\
\hline
\end{tabular}

*P $<0,01$ comparado com o grupo $\mathrm{B}$.

De acordo com o método empregado na avaliação histológica, a densidade média dos elementos histopatológicos estudados foi de $105 \pm 17,1$ nos animais do grupo A e 146,2 $\pm 8,8$ no grupo $B(p<0,01)$. Os dados relativos ao estudo histopatológico de ambos os grupos encontram-se resumidos na tabela 3. A diferença entre os dois grupos, ocorreu em decorrência, principalmente, da menor densidade de colágeno e de fibroblastos nas feridas dos animais que receberam a nicotina por via subcutânea. 
Tabela 3 - Densidade média dos parâmetros histológicos analisados nos grupos A e B.

\begin{tabular}{c|c|c}
\hline${\text { Rato } \mathbf{n}^{\mathbf{0}}}^{\mathbf{m}}$ & Grupo A (nicotina) & Grupo B (controle) \\
\hline 01 & 110.4 & 147.0 \\
\hline 02 & 89.0 & 136.0 \\
\hline 03 & 102.9 & 142.0 \\
\hline 04 & 96.3 & 162.5 \\
\hline 05 & 120.9 & 140.1 \\
\hline 06 & 109.5 & 138.8 \\
\hline 07 & 80.7 & 147.6 \\
\hline 08 & 134.0 & 155.0 \\
\hline Média $\pm \mathrm{dp}$ & $105,4 \pm 17,1^{*}$ & $146,2 \pm 8,8$ \\
\hline
\end{tabular}

$* \mathrm{P}<0,01$ comparado com o grupo $\mathrm{B}$

\section{DISCUSSÃO}

A análise da literatura induz à conclusão de que o hábito de fumar leva a uma série de alterações em diversos órgãos, em especial atingindo os pulmões e coração ${ }^{2}$. No que diz respeito à cicatrização das feridas, são poucos os estudos específicos a respeito. Moosa et $\mathrm{al}^{3}$ relatam que os fumantes têm risco aumentado de apresentarem deiscência de feridas da parede abdominal, e Goodson et $\mathrm{al}^{7}$ demonstraram que ocorre uma deposição deficiente de colágeno nas feridas operatórias de fumantes, o que corrobora com os achados do nosso trabalho. Os efeitos vasoconstrictores observados nos fumantes, que são principalmente devidos à nicotina, foram descritos por Lampson' 9 . Os problemas observados nas feridas dos animais do presente estudo certamente foram decorrentes da diminuição da PO2 tecidual nos que usaram nicotina subcutânea, uma vez que esse parâmetro é de suma importância para todos os fenômenos do processo da cicatrizaçãa ${ }^{10,11}$.

Segundo Niinikoski ${ }^{12}$, o fator central e básico do qual depende a realização, a bom termo, de todos os outros fenômenos da cicatrização é o suprimento de oxigênio aos tecidos. Com o auxílio de microeletrodos, foi demonstrado que a tensão de oxigênio próximo aos capilares da ferida estava em torno de 60 a $90 \mathrm{mmHg}$ e, a pequena distância, os valores se aproximavam de zero. Verificou-se, ainda, que os fibroblastos necessitam de uma tensão de oxigênio de $15 \mathrm{mmHg}$ para a divisão celular e de 15 a $30 \mathrm{mmHg}$, para a síntese de coláge$\mathrm{no}^{13}$. Estes mesmos autores ${ }^{12,13}$ observaram que se houver uma diminuição de volume sanguíneo até níveis que provoquem hipotensão arterial, a perfusão de oxigênio da ferida cessa quase inteiramente por longos períodos, interferindo drasticamente na síntese do colágeno. Quanto mais oxigênio houver nos tecidos maior será a síntese do colágeno e mais eficiente será sua maturação ${ }^{14}$. Estes achados demonstram claramente que a vascularização, o teor de oxigênio tecidual e a proteína do colágeno maduro e estável representam fatores de grande importância na cicatrização das feridas.

Foi demonstrado que a nicotina injetada por via subcutânea prejudica a viabilidade de retalhos cutâneos em ratos, aumentando a área de necrose, quando se utiliza a droga no pré-operatório e que a continuidade do uso no pós-operatório aumenta a área comprometida ${ }^{15}$.

O montante de deposição do colágeno, epitelização, angiogênese, culminando com a maturação do colágeno, todos dependem da oxigenação tecidual. No que diz respeito à ação da nicotina na cicatrização da parede abdominal no presente trabalho, é provável que outros mecanismos ainda não bem esclarecidos estejam envolvidos. Tanto o ato de fumar como a aplicação de emplastros de nicotina na pele de pacientes, inibem o fluxo de oxigênio para os tecidos em cicatrização, via vasoconstricção simpaticomimética. Da mesma maneira, o fumo eleva os níveis de carboxihemoglobina no sangue, resultando igualmente em menor tensão de oxigênio nos tecidos. Estudo experimental demonstrou que, mesmo pequenas diminuições de tensão de oxigênio nos tecidos resultam em graves alterações na cicatrização das feridas, com substancial aumento nas taxas de infecção ${ }^{16}$. Rees et $\mathrm{al}^{4}$ documentaram bem um número razoável de complicações da ferida operatória, de retalhos cutâneos e demais localizações, todas decorrentes do uso do tabaco, tanto no homem como em animais de laboratório.

A determinação da resistência da camada musculoaponeurótica foi testada com a mesma metodologia do presente trabalho, com resultados semelhantes ${ }^{17}$. Entretanto, o dinamômetro e outros equipamentos têm sido utilizados para a aferição da resistência dos tecidos biológicos $^{18}$. 


\section{CONCLUSÃO}

Com base nos resultados encontrados podemos concluir que a nicotina por via subcutânea, em ratos, exerce efeito deletério sobre a cicatrização de lesões da parede abdominal quanto à $\mathrm{pO}_{2}$ dos tecidos em cicatrização, resistência à tensão e histopatologia.

\section{REFERÊNCIAS}

1. Benowitz NL. Pharmacologic aspects of cigarette smoking and nicotine addiction. N Engl J Med 1988; 319: 1318-1330.

2. National Research Council, Committee on Passive Smoking. Environmental tobacco smoke: measuring sposures and assessing health effects. Washington, DC: National Academic Press 1986.

3. Moosa AR, Jones ML, Scott M. Surgical complications. In: Sabiston DC, ed. Textbook of Surgery. Philadelphia, PA: WB Saunders Co; 1986: 340.

4. Rees TC, Liverett DM, Guy CI. The effect of cigarette smoking on skin flap survival in the face lift patient. Plast Reconstr Surg 1984; 73: 911-915.

5. OReifkohl R, Wolfe JÁ, Cox EB, McCarty KS. Association between cutaneous occlusive vascular disease, cigarette smoking and skin slough after rhytidectomy. Plast Reconstr Surg 1986: 77: 592-595.

6. Mosely L, Finseth F. Cigarette smoking: impairement of digital blood flow in the hand. Hand 1977; 98: 97-101.

7. Goodson III WH, Hunt TK. Wound healing in well-controled diabetic men. Surg Forum 1984; 35: 614-616.
8. Aydos RD, Magalhães AM, Menezes Filho JF, Barone B, Goldenberg S. Efeitos da diatermia cirúrgica na cicatrização de anastomoses do intestino grosso. Estudo experimental em coelhos. Acta Cir Bras 1994;9:190-194.

9. Lampson RS. Quantitative study of the vasoconstriction induced by smoking. JAMA 1935; 104: 1964-1966.

10. Chvapil M, Hurych J, Ehrichova E. The influence of varying oxigen tensions uppon proline hydroxylation and the metabolism of collagenous and noncollagenos proteins in the slices. Z Physiol Chem 1968; 349: 211-217.

11. Prockop D, Kivirikko KI, Tudrman L, Guszman NA. The biosynthesis of collagen and its disorders. N Engl J Med 1979; 301: 13-23

12. Niinikoski J. Effect of oxigen supply on wound healing tissues. Acta Psiol Scand, sup. 334, 1969

13 Niinikoski J, Hunt TK, Dunphy JE. Oxigen supply in healing tissues. Am J Surg 1972; 123: 247.

14. Kivisaari J, Viheesaari T, Renvall S, Niinikoski J. Energy metabolism of experimental wounds at various oxigen environments. Ann Surg 1975; 181: 823-827.

15. Campos H, Ferreira LM, Santos WLC, Araújo MCM. Efeitos da nicotina nos retalhos cutâneos em ratos. Acta Cir Bras 2001; 16:206-210.

16. LaVan FB, Hunt TK. Oxigen and wound healing. Clin Plast Surg 1990;17:463-466.

17. Tognini JRF, Goldenberg S, Naresse LE, Simões MJ, Alves FLG, Magalhães AM. Estudo comparativo entre a sutura contínua e a com pontos separados na parede abdominal de ratos. Acta Cir Bras 1997; 12:249-254.

18. Westphalen ACA, Cunha MEP, Camargo PV, Silva RC, Teruschkin M, Edelweiss MIA, Gomes C. Tendão x cicatrização retomada da resistência. Acta Cir Bras 1995;10:169-172.

Medeiros AC, Lima FP, Dantas Filho AM, Melo NMC, Azevêdo IM. The nicotine has a deleterious effect on the healing of abdominal wall. Acta Cir Bras [online] 2003 vol 18 suppl 1. Available in www.scielo.br/acb.

ABSTRACT Objetive: An experimental study was done to evaluate the effect of nicotine on the healing of abdominal wall. Methods: Sexteen Wistar rats weighing $210 \pm 8 \mathrm{~g}$ were randomly separated into two groups of eight rats each. In the group A Nicotine (Nicotinel O) was implanted in the back subcutaneous $5 \mathrm{mg} / \mathrm{Kg}$ each two days, begining at the fifth preoperative day. A $5 \mathrm{~cm}$ median laparotomy was done, sutured with nylon 5-0 and the rats were observed in individual cages. The group B (control) didn't use nicotine. A multiperfurated silicone tube was implanted subcutaneously near the abdominal sutured lesion. In the tenth postoperative day $1 \mathrm{ml}$ of serous liquid was harvested from the silicone tube and a $\mathrm{pO}_{2}$ dosage was done. The rats were killed with overdose of anesthetic. A $2 \mathrm{~cm}$ width transversal segment of abdominal wall was submited to tensile strength test by a tensiometer. A biopsy of the sutured area including muscle, aporeurosis and peritoneum was processed for histopatologic analysis by a digitalised system. The data were processed by the ANOVA and Newman-Keuls tests, considering significant the differences when $p<0,05$. Results: In the group $\mathrm{A}$ the $\mathrm{pO}_{2}$ on the tissue serous liquid reached $17,75 \pm 3,4 \mathrm{mmHg}$ and in the group $\mathrm{B}$ (control) the $\mathrm{pO}_{2}=40,75 \pm 6,4 \mathrm{mmHg}(\mathrm{p}<0,01)$. The tensile strength reached $728,5 \pm 161,75 \mathrm{gf}$ in the group $A$ and $1241,6 \pm 232 \mathrm{gf}$ in the control one $(\mathrm{p}<0,01)$ The mean density of the histopatologic elements was $105 \pm 17,1$ in group $A$ and $146,2 \pm 8,8$ in group $B(p<0,01)$.Conclusion: after evaluation of tissue $\mathrm{pO}_{2}$, tensiometry and histopatology, the data permited to conclude that the nicotine has a deleterious effect on the healing the abdominal wall of rats.

KEY WORDS: Nicotine. Healing. Abdominal wall. Tensiometry. Histopatology. Oxigen.

Correspondência:

Aldo da Cunha Medeiros

Av. Miguel Alcides Araújo 1889 - 59078-270 Natal-RN 